\title{
Estimation of blood glucose concentration during endurance sports
}

\author{
Giovanni Sebastiani ${ }^{1,2,4}$, Stig Uteng ${ }^{3}$, Fred Godtliebsen ${ }^{4}$, Jan Polàk ${ }^{5}$ and Jan Brož ${ }^{6}$, \\ 1) Istituto per le Applicazioni del Calcolo "Mauro Picone", CNR, Rome, Italy, \\ 2) Department of Mathematics "Guido Castelnuovo", "Sapienza University of Rome", Italy, \\ 3) Department of Education UIT The Arctic University of Norway, Tromsø, Norway, \\ 4) Department of Mathematics and Statistics UIT The Arctic University of Norway, Troms $\varnothing$, Norway, \\ 5) Department of Pathophysiology, Third Faculty of Medicine, Charles University, Prague, Czech Republic, \\ 6) Department of Internal Medicine, Second Faculty of Medicine, Charles University, Prague, Czech Republic
}

Received: May 22, 2020. Revised: June 19, 2020. Accepted: July 9, 2020. Published: July 21, 2020.

\begin{abstract}
In this paper, we describe a new statistical approach to estimate blood glucose concentration along time during endurance sports based on measurements of glucose concentration in subcutaneous interstitial tissue. The final goal is the monitoring of glucose concentration in blood to maximize performance in endurance sports. Blood glucose concentration control during and after aerobic physical activity could also be useful to reduce the risk of hypoglycemia in type 1 diabetes mellitus subjects. By means of a low invasive technology known as "continuous glucose monitoring", glucose concentration in subcutaneous interstitial tissue can now be measured every five minutes. However, it can be expressed as function of blood glucose concentration along time by means of a convolution integral equation. In the training phase of the proposed approach, based on measurements of glucose concentration in both artery and subcutaneous interstitial tissue during physical activity, the parameters of the convolution kernel are estimated. Then, given a new subject performing aerobic physical activity, a deconvolution problem is solved to estimate glucose concentration in blood from continuous glucose monitoring measurements.
\end{abstract}

Keywords- Endurance sports, blood glucose concentration, continuous glucose monitoring

\section{INTRODUCTION}

$\mathbf{P}$ HYSICAL activity (PA) in endurance sports is characterized by prolonged muscular work at high intensity (high heart frequency). The energy needed to maintain muscular fiber contraction during endurance sports is mainly produced by means of chemical transformations of fatty acids and glucose. Although energy production obtained from lipids is necessary in endurance PA, glucose as source of energy plays a very important role. Glucose is stored in the human body in muscles and liver in the form of glycogen, and several grams of glucose are diluted in blood. During PA, glucose is produced in muscles by glycogenolysis. In addition, it is also transported there by means of blood circulation: the sources are liver glycogenolysis, gluconeogenesis in liver and kidneys and ingested food [11]. Demand of glucose in high intensity exercise is substantial and could potentially lead to hypoglycemia, i.e. drop of blood glucose (BG) concentration below $3.6 \mathrm{mmol} / \mathrm{l}$ (glycemia) [10]. Thus, to keep glycemia within the normal range (3.6-5.6 mmol/l) (see [10]) the influx of glucose from blood to the muscles is regulated by several hormons among which insulin plays the major role. Insulin production is decreased during exercise to prevent excessive leak of glucose into the muscles [17]. From the above considerations, it is clear that monitoring glucose concentration in tissues and blood can be very relevant to maximize performance in endurance sports. Moreover, it is absolutely essential for subjects with type 1 diabetes mellitus (T1D) in which insuline is not produced and must be injected subcutaneoulsy. Thus, the risk of hypoglycemia in T1D subjects during PA is relevant because the physiological system to minimize it is disrupted. To minimise such a risk it is recommended that the insulin dose administered before and after an exercise period should be decreased, carbohydrates should be ingested regularly and glycemia should be measured as frequently as possible (at least every $30 \mathrm{~min}$ ) [20].

Recently, a new and low invasive technology has been introduced, known as continuous glucose monitoring (CGM), by which glucose concentration in subcutaneous interstitial tissue can be measured every five minutes [15]. This technique is mainly used by T1D subjects, who greatly benefit of it to monitor the level of glucose in tissues and blood in order to control it. Studies proved that T1D patients can significantly benefit of regular aerobic PA, which reduces glucose concentration in both blood and tissues [4]. Unfortunately, the fear of the potentially severe effects of getting hypoglycemia during or after performing PA is a major reason why most T1D subjects do not perform PA [3].

We point out that by CGM we can only measure glucose concentration in subcutaneous interstitial tissue $G_{s}(t)$ at sampling times while we are interested more in temporal glucose level in tissues and blood. Fast and large changes in glucose concentration in blood $G_{b}(t)$, can be observed during PA. However, it is well known that related changes in $G_{s}(t)$ are seen after a time delay (lag time) [0], [23]. The quantity $G_{s}(t)$ can be modeled as a function of $G_{b}(t)$ by means of a 
convolution integral equation with asymmetric kernel involving only the past due to causality [6].

Here, we focus on the estimation of glucose concentration dynamics in blood during aerobic PA from CGM measurements. To this aim, we propose to first perform some PA experiments on one or more subjects. During training, CGM measurements are performed together with those of glucose concentration in both artery and capillaries, that reveal to be very close to each other. These data are then used to estimate the convolution kernel parameters. Given a new subject, a deconvolution problem is then solved to estimate $G_{b}(t)$ from CGM measurements during PA. This task could be introduced within a general tool, which also includes glucose concentration forecasting either by machine learning or by mathematical models [1], in order to monitor glucose concentration in blood aiming to maximize performance in endurance sports. In connection to the control of BG concentration during PA and after it could also be useful to reduce the risk of hypoglycemia in T1D subjects, and recent work, [2, 7, 8, 12, 13, 16, 18, 19, 21, 24], indicate the importance of handling PA for T1D patients. The current paper makes progress in this direction by obtaining a more precise description of BG levels in artery. Utilizing this new information may be the key to better BG control during PA.

Here we present initial results that indicate that the wellknown delay in CGM measurements can be significantly reduced. This delay may be life threatening for T1D patients, in particular during PA where the BG level can be lowered rapidly. Future utilization of these results are therefore expected to improve the BG behavior in T1D patients in general and in particular during PA.

\section{PA EXPERIMENTS AND MODELS}

PA experiments are performed using an electrically braked ergometer at the target heart rate (THR) according to an intensity $I$ corresponding to $50 \%$ of individual heart rate reservoir, calculated following the Karvonen equation: $T H R=\left(H R_{\max }-H R_{\text {rest }}\right) * I / 100+H R_{\text {rest }}$ [14]. Measurements of CGM and glucose concentration in artery and capillaries are performed every 5 and 10-15 minutes, respectively both during PA and for 60 minutes after its end. The training begins approximately 120 minutes since breakfast and after 10 minutes warm up phase. The exercise is interrupted when either symptoms of hypoglycemia occurred or $\mathrm{BG}$ concentration of $3.5 \mathrm{mmol} / \mathrm{l}$ is measured. Immediately at end of training, $20 \mathrm{~g}$ of glucose diluted in $150 \mathrm{ml}$ of water is ingested.

We focus here on experiments with a PA phase followed at its end by glucose solution ingestion. To describe both $G_{b}(t)$ and $G_{s}(t)$ we therefore adopted a model with two sine functions. The first sine function decreases until its local minimum is reached. Then, the second sine function increases starting from this point which corresponds to its local minimum. This ensures that also right and left derivatives at common local minimum point are equal. The model used has the following parameters: minimum location, 3 parameters for each sine function, but only six of them are free because of the continuity constraint of the two sine functions at their local minimum point.
The quantity $G_{s}(t)$ as a function of $G_{b}(t)$ is commonly modeled [6] by means of asymmetric convolution, due to causality, with exponential kernel $h(t)$ :

$$
G_{s}(t)=\int_{0}^{t} G_{b}(\tau) h(t-\tau) d \tau+\epsilon, \epsilon \sim N\left(0, \sigma^{2}\right)
$$

which is discretized to:

$$
\mathbf{G}_{s}=\mathbf{H G}_{b}+\epsilon, \epsilon \sim N\left(0, \sigma^{2} \mathbf{I}\right) .
$$

However, due to the two phases of glucose decrease and increase with different dynamics, here we use two different exponential functions for the convolution kernel, one before the minimum location of $G_{b}(t)$ and the other one after it. Given the functions $G_{b}(t)$ and $G_{s}(t)$ estimated for one subject or more, the convolution kernel parameters are estimated by minimizing the mean squared error between $G_{s}(t)$ and its estimate by numerical convolution of $G_{b}(t)$. In a new subject, model parameters of $G_{s}(t)$ are estimated from CGM measurements by minimizing mean squared error between data and model values at sampling times. Deconvolution of $G_{s}(t)$ is then performed to estimate model parameters for $G_{b}(t)$ by minimization of mean squared errors between estimated $G_{s}(t)$ and the numerical convolution of $G_{b}(t)$. Here the convolution kernel is assumed to be known since we use the one already estimated before. This minimization is performed here by Simulated Annealing, which will be the dominating algorithm in terms of complexity, $O\left(k^{5}\right)$, as given in [22], where $k$ is the length of the data vector for each patient. The complexity of least square estimation is $O\left(k^{3}\right)$, due to matrix inversion and for the convolution (i.e. matrix-vector multiplication), $O\left(k^{2}\right)$, both given in [5]. The method is summarized

\begin{tabular}{|c|c|}
\hline \multicolumn{2}{|c|}{$\begin{array}{ll}\text { Algorithm } 1 \text { The CGMPA algorithm } & \text { The }\end{array}$} \\
\hline $\begin{array}{l}\text { Input: } \\
\text { centration } \\
\text { Training: }\end{array}$ & Measurements of CGM and arterial glucose con- \\
\hline & $\begin{array}{l}\text { For } i=1: n \\
\quad \text { Model fitting to CGM data of subject } \mathrm{i} \\
\quad \text { Model fitting to arterial data of subject } \mathrm{i}\end{array}$ \\
\hline & End \\
\hline Estimation: & Kernel parameters via convolution \\
\hline Input: & CGM measurements for a new subject \\
\hline Estimation: & $\begin{array}{l}G_{s}(t) \text { parameters via CGM data fitting } \\
G_{b}(t) \text { parameters via deconvolution of } G_{s}(t)\end{array}$ \\
\hline
\end{tabular}
in Algorithm (1).

\section{RESULTS}

In Fig. 1 we can observe the data of both CGM and arterial glucose concentration measured for one subject during the PA experiment. A double sine model is fitted by minimizing the mean square error for both the $G_{s}(t)$-data and the $G_{b}(t)$-data. As seen, there is a very good agreement between model and data. In the initial phase, $G_{b}(t)$ decreases due to PA. The ingestion of a glucose solution at the end of PA, makes $G_{b}(t)$ to increase again. The changes of $G_{s}(t)$ due first to PA and then to glucose ingestion appear clearly delayed w.r.t. those 


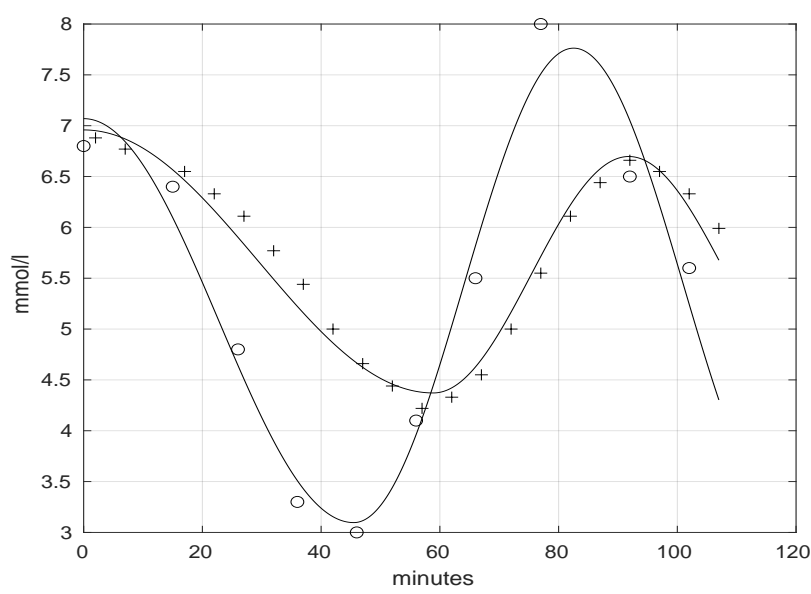

Fig. 1: Example of data for CGM (+) and arterial glucose concentration (o) measured for one subject during PA experiment. Continuous lines show best data fits by double sine model for $G_{s}(t)$ and $G_{b}(t)$, respectively.

of $G_{b}(t)$. This can be claimed since it is clear, for e.g. the upper panel of Fig. 2, that the variation in CGM is delayed compared to BG in artery during both PA and after intake of glucose at the end of PA. To see this, draw a horizontal line for e.g. a value of $8 \mathrm{mmol} / \mathrm{l}$. During PA, $G_{b}(t)$ arrives to this value after around 35 minutes while $G_{s}(t)$ arrives to this value after around 50 minutes. This means a delay of around 15 minutes, a length that may be life threatening for a T1D patient. The same behavior for this horizontal line is observed during the glucose ingestion where $G_{b}(t)$ arrives to $8 \mathrm{mmol} / \mathrm{l}$ after around 95 minutes while $G_{s}(t)$ arrives there after 110 minutes. Note that also here the delay is around 15 minutes.

Given the functions $G_{b}(t)$ and $G_{s}(t)$ estimated for this subject, the convolution kernel parameters are estimated by minimizing the mean squared error between $G_{s}(t)$ and its estimate by numerical convolution of $G_{b}(t)$. This is done by exhaustive search on a finite $2 \mathrm{~d}$-grid of values for the time constants (in minutes) of the two exponential functions from 1 minute to the maximum value of sampling times (in minutes) with steps of 1 minute.

Fig. 2 shows similar data of CGM and arterial glucose concentration for two other subjects. The best model fit for $G_{b}(t)$ is superimposed on the arterial glucose concentration data. Based on the convolution kernel estimated before, an estimate for $G_{S}(t)$ is obtained by numerical convolution of $G_{b}(t)$. A good agreement is observed between CGM data and estimated $G_{s}(t)$ computed at sampling times.

In Fig. 3 the same data of CGM and arterial glucose concentration as in Fig. 2 appear. The best model fit for $G_{s}(t)$ is superimposed on the CGM data. Based on the convolution kernel estimated before, an estimate for $G_{b}(t)$ is obtained by numerical deconvolution of $G_{s}(t)$. A good agreement is observed between arterial glucose concentration data and estimated $G_{b}(t)$ computed at sampling times.

The percentage total mean square error for the mean square error minimization fitting for the convolution and de-
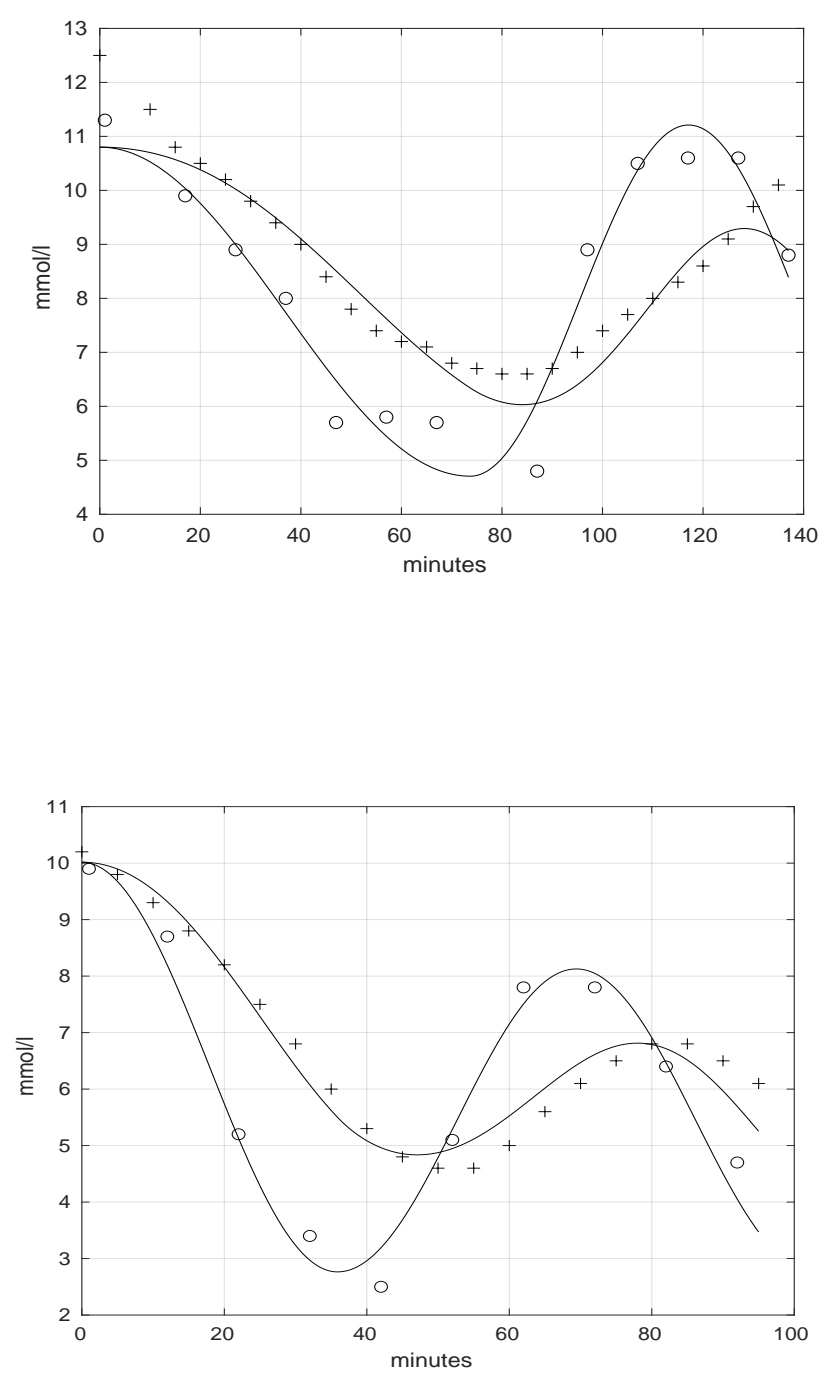

Fig. 2: Data for CGM (+) and arterial glucose concentration (o) measured for two additional persons performing a similar PA experiment. The best model for $G_{b}(t)$ fitted to arterial glucose concentration data is also superimposed on them. For each of the two subjects, estimate of $G_{s}(t)$, as obtained by numerical convolution of $G_{b}(t)$, is superimposed on the CGM data. 

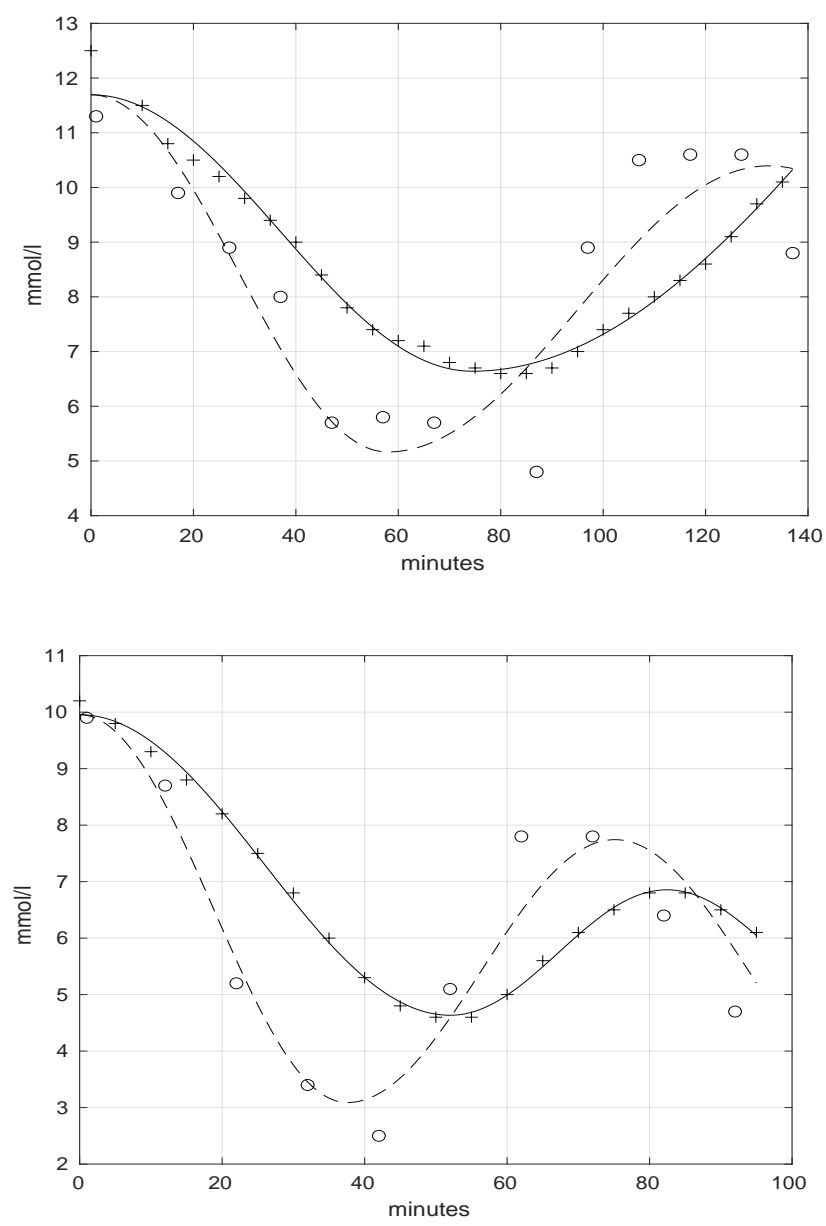

Fig. 3: Data for CGM (+) and arterial glucose concentration (o) as in Fig. 2 Best model for $G_{s}(t)$ fitted to CGM data is also superimposed on them. For each of the two subjects, estimate of $G_{b}(t)$ obtained by numerical deconvolution of $G_{s}(t)$ is superimposed on the arterial glucose concentration data.

convolution estimates for the two subjects in Figs. 2 and 3 are shown in Table 1 .

\begin{tabular}{ccc}
\hline Subject & Convolution & Deconvolution \\
\hline 1 & $G_{b}: 7 \% G_{s}: 5 \%$ & $G_{s}: 2 \% G_{b}: 10 \%$ \\
2 & $G_{b}: 6 \% G_{s}: 5 \%$ & $G_{s}: 0.3 \% G_{b}: 11 \%$ \\
\hline
\end{tabular}

Table 1: Percentage total mean square error for the mean square error minimization fitting for the convolution and deconvolution estimates for the two subjects in Figs. 2 and 3

\section{CONCLUSION}

The present work shows that a statistical approach can be used to successfully estimate BG concentration along time during PA from CGM measurements. This task could be introduced within a general tool to monitor glucose concentration in blood aiming to maximize performance in endurance sports. This could also be used to reduce the risk of hypoglycemia in T1D subjects performing PA.

\section{REFERENCES}

[1] Ajmera, I., Swat, M., Laibe, C., Novére, N. L., and Chelliah, V. (2013). The impact of mathematical modeling on the understanding of diabetes and related complications. CPT: Pharmacometrics and Systems Pharmacology, 2(e54).

[2] Beneyto, A., Bertachi, A., Bondia, J., and Vehi, J. (2020). A New Blood Glucose Control Scheme for Unannounced Exercise in Type 1 Diabetic Subjects. IEEE Transactions on Control Systems Technology, 28(2):593-600.

[3] Brazeau, A.-S., Rabasa-Lhoret, R., Strychar, I., and Mircescu, H. (2008). Barriers to Physical Activity Among Patients With Type 1 Diabetes. Diabetes Care, 31(11):2108-2109.

[4] Chimen, M., Kennedy, A., Nirantharakumar, K., Pang, T. T., Andrews, R., and Narendran, P. (2012). What are the health benefits of physical activity in type 1 diabetes mellitus? A literature review. Diabetologia, 55(3):542551.

[5] Cormen, T. H., Leiserson, C. E., and Rivest, R. L. (2009). Introduction to Algorithms, Second Edition. MIT Press.

[6] Dicker, L. H., Sun, T., Zhang, C. H., Keenan, D. B., and Shepp, L. (2013). Continuous blood glucose monitoring: A Bayes-hidden Markov approach. Statistica Sinica, 23(4):1595-1627.

[7] Frank, S., Jbaily, A., Hinshaw, L., Basu, R., Basu, A., and Szeri, A. J. (2018). Modeling the acute effects of exercise on insulin kinetics in type 1 diabetes. Journal of Pharmacokinetics and Pharmacodynamics, 45(6):829-845.

[8] Garcia-Tirado, J., Corbett, J. P., Boiroux, D., Jørgensen, J. B., and Breton, M. D. (2019). Closed-loop control with unannounced exercise for adults with type 1 diabetes using the Ensemble Model Predictive Control. Journal of Process Control, 80:202-210.

[9] Garg, S. K., Voelmle, M., and Gottlieb, P. A. (2010). Time lag characterization of two continuous glucose monitoring systems. Diabetes Research and Clinical Practice, 87(3):348-353.

[10] Güemes, M., Rahman, S. A., and Hussain, K. (2016). What is a normal blood glucose? Archives of Disease in Childhood, 101(6):569-574.

[11] Hargreaves, M. (2000). Skeletal muscle metabolism during exercise in humans. Clinical and Experimental Pharmacology and Physiology, 27(3):225-228.

[12] Hobbs, N., Hajizadeh, I., Rashid, M., Turksoy, K., Breton, M., and Cinar, A. (2019). Improving Glucose Prediction Accuracy in Physically Active Adolescents With Type 1 Diabetes. Journal of Diabetes Science and Technology, 13(4):718-727. 
[13] Jaggers, J. R., King, K. M., Watson, S. E., and Wintergerst, K. A. (2019). Predicting Nocturnal Hypoglycemia with Measures of Physical Activity Intensity in Adolescent Athletes with Type 1 Diabetes. Diabetes Technology and Therapeutics, 21(7):406-408.

[14] Karvonen, M. J., Kentala, E., and Mustala, O. (1957). The effects of training on heart rate; a longitudinal study. Annales medicinae experimentalis et biologiae Fenniae, 35(3):307-315.

[15] Mian, Z., Hermayer, K. L., and Jenkins, A. (2019). Continuous Glucose Monitoring: Review of an Innovation in Diabetes Management. American Journal of the Medical Sciences, 5(5):377-390.

[16] Paoletti, N., Liu, K. S., Smolka, S. A., and Lin, S. (2017). Data-driven robust control for type 1 diabetes under meal and exercise uncertainties. In Lecture Notes in Computer Science (including subseries Lecture Notes in Artificial Intelligence and Lecture Notes in Bioinformatics), volume 10545, pages 23-39.

[17] Raguso, C. A., Coggan, A. R., Gastaldelli, A., Sidossis, L. S., Bastyr, E. J., and Wolfe, R. R. (1995). Lipid and carbohydrate metabolism in IDDM during moderate and intense exercise. Diabetes, 44(9):1066-1074.

[18] Resalat, N., Youssef, J. E., Reddy, R., and Jacobs, P. G. (2016). Design of a dual-hormone model predictive control for artificial pancreas with exercise model. In Proceedings of the Annual International Conference of the IEEE Engineering in Medicine and Biology Society, EMBS, pages 2270-2273.

[19] Resalat, N., Youssef, J. E., Reddy, R., and Jacobs, P. G. (2017). Evaluation of model complexity in model predictive control within an exercise-enabled artificial pancreas. IFAC-PapersOnLine, 50(1):7756-7761.

[20] Riddell, M. C., Gallen, I. W., Smart, C. E., Taplin, C. E., Adolfsson, P., Lumb, A. N., Kowalski, A., Rabasa-Lhoret, R., McCrimmon, R. J., Hume, C., Annan, F., Fournier, P. A., Graham, C., Bode, B., Galassetti, P., Jones, T. W., Millán, I. S., Heise, T., Peters, A. L., Petz, A., and Laffel, L. M. (2017). Exercise management in type 1 diabetes: a consensus statement. The Lancet Diabetes and Endocrinology, 55(3):542-551.

[21] Riddell, M. C., Zaharieva, D. P., Tansey, M., Tsalikian, E., Admon, G., Li, Z., Kollman, C., and Beck, R. W. (2019). Individual glucose responses to prolonged moderate intensity aerobic exercise in adolescents with type 1 diabetes: The higher they start, the harder they fall. Pediatric Diabetes, 20(1):99-106.

[22] Sasaki, G. H. and Hajek, B. (1988). The time complexity of maximum matching by simulated annealing. Journal of the ACM (JACM), 35:387-403.

[23] Wei, C., Lunn, D. J., Acerini, C. L., Allen, J. M., Larsen, A. M., Wilinska, M. E., Dunger, D. B., and Hovorka, R. (2010). Measurement delay associated with the
Guardian $\AA$ RT continuous glucose monitoring system. Diabetic Medicine, 27(1):117-122.

[24] Yardley, J. E. (2020). Exercise and the Artificial Pancreas: Trying to Predict the Unpredictable in Patients With Type 1 Diabetes? Canadian Journal of Diabetes, 44:119120.

\section{Creative Commons Attribution License 4.0 (Attribution 4.0 International, CC BY 4.0)}

This article is published under the terms of the Creative Commons Attribution License 4.0 https://creativecommons.org/licenses/by/4.0/deed.en_US 